\title{
A Case of Spontaneous Temporomandibular Joint Herniation into the External Auditory Canal with Clicking Sound
}

\author{
Tae Hyun Kim, Sun Kyu Lee, Su Jin Kim and Jae Yong Byun \\ Department of Otorhinolaryngology-Head and Neck Surgery, Kyung Hee University Hospital at Gangdong, Seoul, Korea
}

$\begin{array}{ll}\text { Received } & \text { March 27, } 2013 \\ \text { Revised } & \text { April 11, 2013 } \\ \text { Accepted } & \text { May 12, 2013 }\end{array}$

Address for correspondence Jae Yong Byun, MD, PhD

Department of OtorhinolaryngologyHead and Neck Surgery, Kyung Hee University Hospital at Gangdong, 892 Dongnam-ro, Gangdong-gu,

Seoul 134-727, Korea

Tel $+82-2-440-8160$

Fax $+82-2-440-8470$

E-mail otorhino512@naver.com
A bony defect of the external auditory canal (EAC) and herniation of the temporomandibular joint (TMJ) can be caused by tumorous conditions, inflammation, trauma, and otologic procedures. Spontaneous TMJ herniation into the EAC can be caused by a congenital bony defect known as patent Huschke's foramen, which is a very rare condition. In our case, an objective clicking sound was produced when the patient opened his mouth. A protruding mass was found in the anterior wall of the EAC during mouth closing, and herniation of the TMJ was confirmed with computed tomography. Therefore, we thought the clicking sound of our case could have resulted from spontaneous TMJ herniation through the patent foramen of Huschke.

Korean J Audiol 2013;17:90-93

KEY WORDS: Huschke's foramen · Temporomandibular joint · Tinnitus.

\section{Introduction}

The external auditory canal (EAC) is intimately related to the temporomandibular joint (TMJ), separated only by its bony anterior wall, which is of variable thickness. The herniation of the TMJ which is protruded into the EAC is mostly known to occur from tumor, injury, and inflammation. On the other hand, spontaneous TMJ herniation resulting from the congenital bony defect is a very rare condition, and it is assumed this is caused by a persistent foramen of Huschke.

During the osseous development of the tympanic bone, the initial osseous EAC was constructed by the fusing of two prominences that arose from the anterior and posterior portions of the tympanic ring. The anterior and posterior processes are growing in a ' $U$ ' shape. This fusion divides the primordial annulus into the superior EAC and inferior Huschke's foramen. The Huschke's foramen is becomes smaller as the tympanic plate grows through a membranous ossification process and usually closes before the age of 5 years. ${ }^{1)}$ But persistent foramen of Huschke is occasionally generated by an imperfect serial fusion process. ${ }^{2)}$

Recently, the authors report a case of a persistent foramen of Huschke and subsequent protrusion of temporomandibular joint tissue into the EAC in a patient who visited our hospi- tal for the chief complaint of clicking tinnitus.

\section{Case Report}

A 53-year-old man came to the Otorhinolaryngology department as an outpatient in September 2011 for the chief complaint of clicking tinnitus on the left. The patient had a past medical history of arrhythmia and heart valve disease but there was no history of trauma or surgery which could have led to tinnitus. Since July 2008, he had received rehabilitation treatment due to cerebral infarction in the middle cerebral artery zone. The clicking tinnitus started suddenly 3 weeks before he visited the hospital, mostly during mastication and when opening and closing his mouth. During the 3 weeks, the clicking tinnitus happened without strength change and without other otologic symptoms such as otalgia and otorrhea. In the otoscopic examination, the tympanic membrane was normal but there was slight protrusion of the soft tissue, originating from the anterior wall of the left EAC. This protrusion was observed when the mouth was closed and if it was open, the protrusion retracted (Fig. 1). The clicking sound that was heard in the left ear of the patient was also heard by the observer when he put a stethoscope on the EAC of the patient when the mouth was opened and closed. 
The patient did not feel uncomfortable in his daily life from the clicking tinnitus, and the Tinnitus Handicap Inventory (THI) score was 18. According to the pure tone audiometry, the air conduction average threshold value (4 division method) was $23 \mathrm{~dB}$ on the right and $22 \mathrm{~dB}$ on the left while the average threshold value from bone conduction audiometry

Fig. 1. A: Otoendoscopic finding shows protruding lesion (white arrow) on anterior wall of left external auditory canal during closing mouth. $\mathrm{B}$ : This lesion was reduced with mouth opening (black arrow).
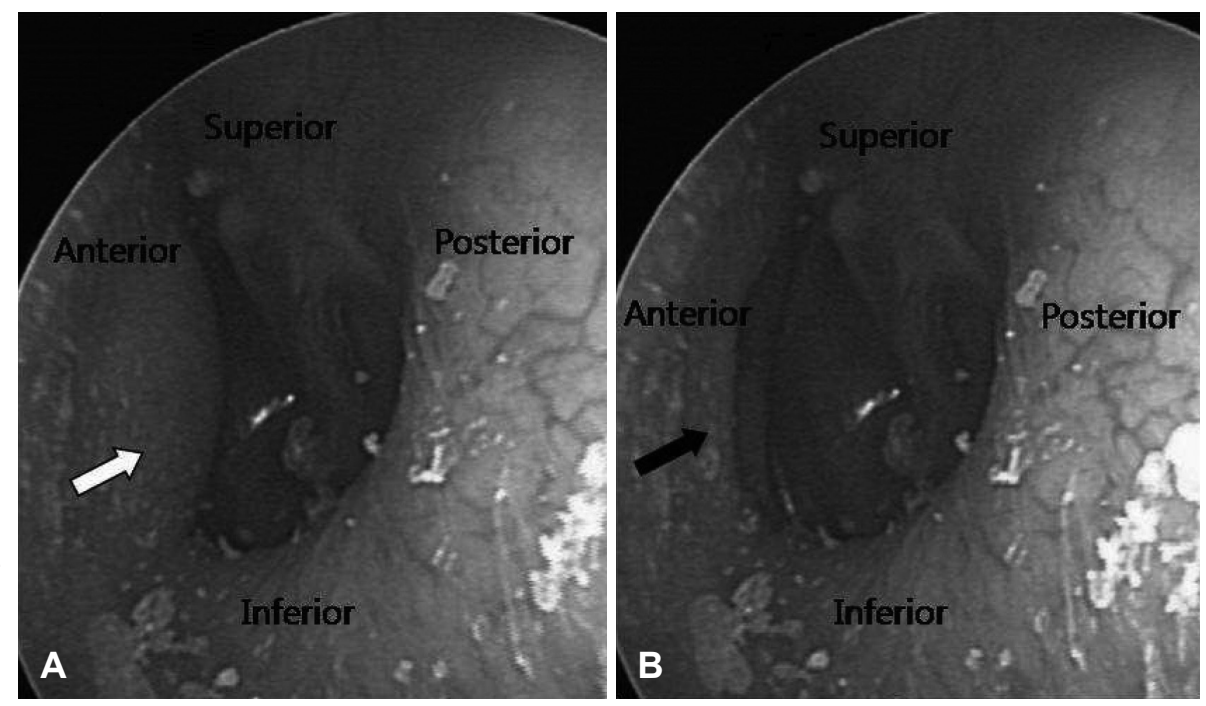

Fig. 2. Temporomandibular joint scalograms. A: Closed mouth view. $B$ : Open mouth view. Note that the mandibular condyle (black arrow) is outside of the glenoid fossa (white arrow).
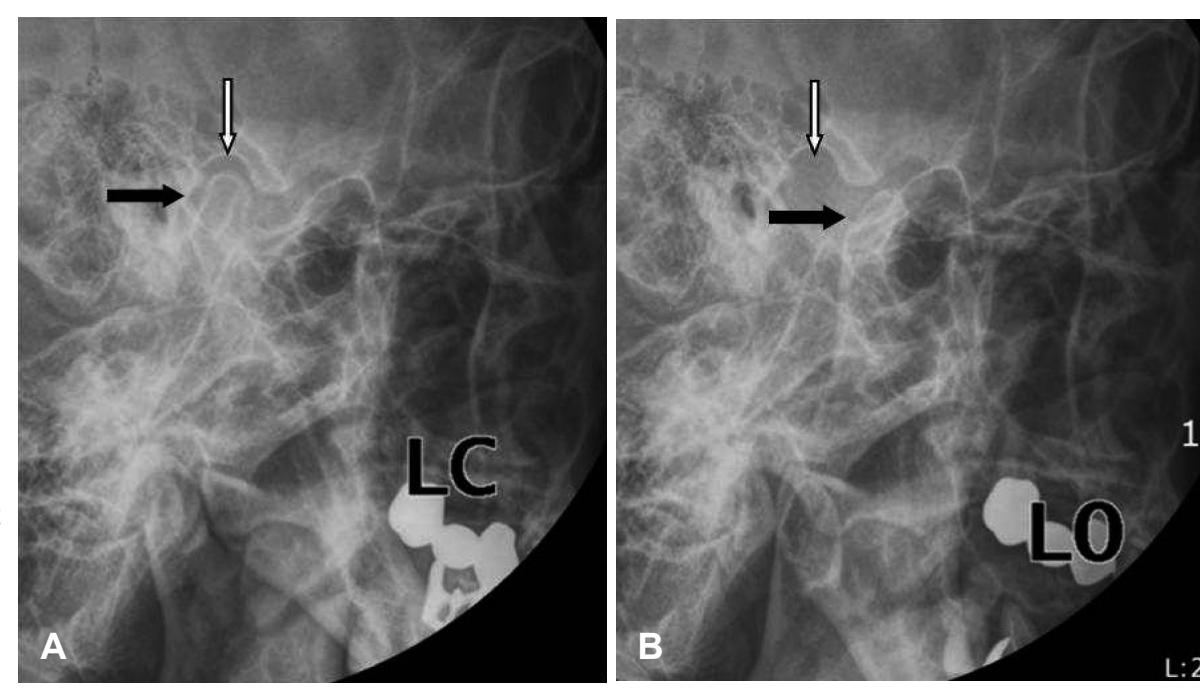

Fig. 3. Temporal bone computed tomography scan showing defect (white arrow) in the anterior bony wall of left external auditory canal. A: Axial view. B: Coronal view.
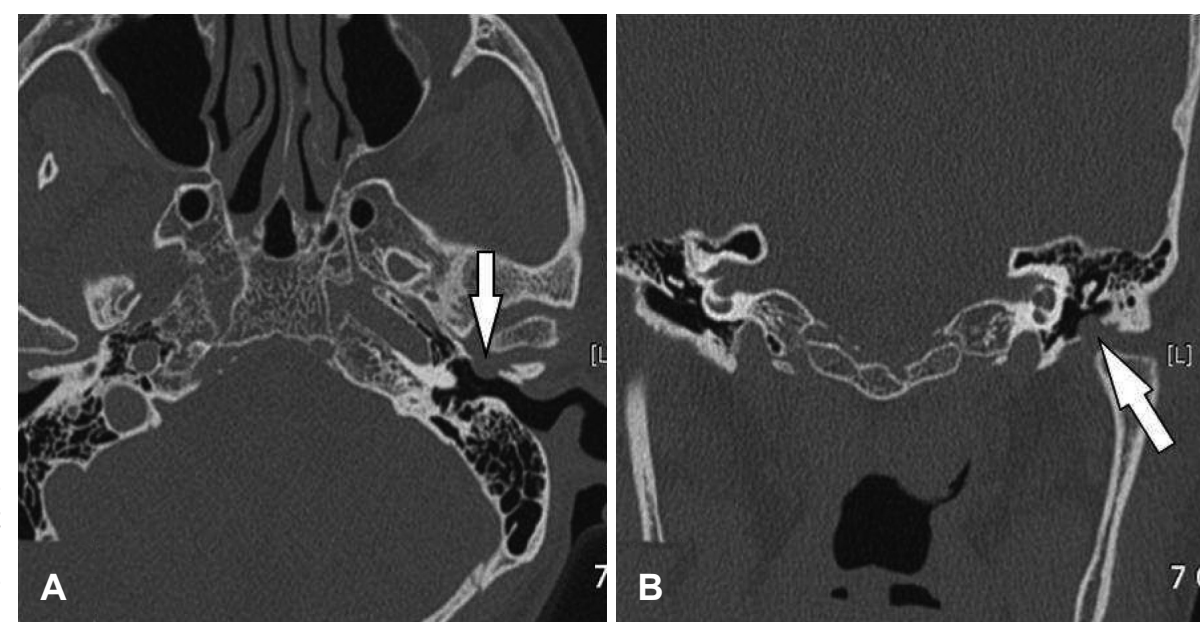
was $21 \mathrm{~dB}$ and $20 \mathrm{~dB}$ respectively. On the impedance test, both sides were A type. We conducted a tinnitogram but the patient complained of just clicking and we failed to find the exact strength and frequency.

According to his otologic history, he had clicking at the time of mastication. Pure tone audiometry also failed to detect sensorineural or conductive hearing loss while the otoscopy found an anterior wall protrusion at the left EAC, and so TMJ herniation was suspected. Therefore, we conducted TMJ X-ray and temporal computed tomography. From the TMJ X-ray, we could see a left temporomandibular joint herniation at the time of mastication (Fig. 2), and the temporal bone computed tomography detected a $6 \mathrm{~mm}$ bony defect in the left EAC anterior wall and subsequent soft tissue herniation (Fig. 3). At the right EAC, there was no bony defect or other unusual findings. The left clicking tinnitus complained by the patient was revealed to occur from TMJ tissue herniation into the EAC at the time of mastication. Therefore, we considered surgical treatment for the left EAC anterior wall defect but the patient refused surgery because he felt uncomfortable with surgical treatment as he was receiving rehabilitation for cerebral infarction, and furthermore, he did not suffer much inconvenience from the clicking tinnitus. Thus, the authors treated him with an anxiolytic and muscle relaxant for 2 weeks, after which we could not see any change in loudness of the tinnitus (according to the patient) and emotional reaction. Now, the authors are observing the progress of the clicking tinnitus.

\section{Discussion}

Tinnitus is an auditory stimulus that is heard without external sound sources, and it can be roughly divided into subjective tinnitus or sensorineural tinnitus, and objective tinnitus or somatosound. Somatosound can be subdivided into muscular origin tinnitus, vascular tinnitus, and patent Eustachian tube origin tinnitus. ${ }^{3)}$ This tinnitus has a lower prevalence rate than sensorineural tinnitus but its cause and occurrence mechanism has been studied well, and the fact that tinnitus can be completely treated with drugs or surgical treatment is important.

The clicking tinnitus in this case was an objective tinnitus that could be detected by the observer by putting a stethoscope on the EAC, as well as by the patient. When we integrated the EAC findings and the radiologic findings, the tinnitus was assumed to be caused by the TMJ herniation. Though the patient was under rehabilitation treatment for cerebral infarction, he had neither a medical history of head and neck surgery or injury nor a medical history of head and neck radiation treatment. Besides, no inflammation was discovered in the EAC on otoscopy, so the cause was more likely to be spontaneous TMJ herniation than secondary TMJ herniation.

The tympanic ring composes a part of the tympanic bone which is undergoing development at the time of birth. During the first year of life, both the anterior process and the posterior process grow toward each other in the tympanic ring for 1 year. At the age of about 1 year, two processes fuse together and a foramen persists medial to the point of fusion. This foramen is called the foramen of Huschke. The foramen of Huschke is occluded slowly as the tympanic plate grows through the membranous ossification process and it is usually considered to be closed fully by 5 years of age. But it occasionally persists, and this is called a persistent foramen of Huschke. ${ }^{4)}$ Lately, the persistent foramen of Huschke is considered a normal variant in temporal bone anatomy, and it is located at the anteroinferior aspect of the EAC, posteromedial to the mandibular head, causing a bony defect of the EAC anterior wall. ${ }^{5)}$

According to previous study, the incidence of persistent foramen of Huschke ranged from $3 \%$ to $24 \%$, and most of the sizes ranged from $1 \mathrm{~mm}$ to $8 \mathrm{~mm}$. A cadaver study reported that the incidence of persistent foramen of Huschke was 7.2\% and the size averaged $2.7 \mathrm{~mm}$ on coronal images and averaged $3.0 \mathrm{~mm}$ on sagittal images. ${ }^{6)}$ The study of Park, et al. ${ }^{7)}$ reported that persistent foramen of Huschke was in 1.5\% of all cases and the TMJ soft tissue herniation was observed in only $26 \%$ of persistent foramen of Huschke cases in spite of the EAC anterior wall defect. In addition, TMJ soft tissue herniation was found to have close a relationship with the size of the defect. In some cases, the initial opening in the Huschke's foreman was probably sufficiently small for no herniation of the TMJ or any other soft tissue into the EAC to have occurred. However, years of mastication could have softened the intervening tissue or enlarged the opening, leading to the observed soft tissue herniation. ${ }^{8)}$

The traumatic influence of masticatory movements may result in enlargement of the defect and the symptoms become more prominent. In symptomatic patients the foramen of Huschke usually reveals itself with otalgia, mandibular joint pain, recurrent infections of the EAC, tinnitus, conductive hearing loss and salivary discharge from the EAC. ${ }^{8)}$ It is known that the condyle itself is not herniated through the foramen of Huschke but retrodiskal tissue or diskal tissue around the TMJ is protruded into the EAC. ${ }^{1,2)}$ The clicking tinnitus in this case occured from the EAC anterior wall movement initiated by the temporomandibular tissue that is herniated by masticatory movements. ${ }^{8,9)}$ In this case, we could also see the EAC anterior wall protruding and retracting whenever the patient opened and closed his mouth respectively, and according to 
the temporal computed tomography, the soft tissue herniated into the EAC.

Treatment of foramen Huschke in symptomatic cases requires closure of the defect in order to prevent the prolapsus of the periarticular tissue into the EAC. There are two kinds of surgical approaches, one is the preauricular approach based on TMJ exposure and the other one is the endaural approach based on anterior exposure of the tympanic bone. ${ }^{8,10)}$ The tragal cartilage for autologous cartilage transplantation is preferable. There have been cases in which tragal cartilage and temporal fascia were used for the repair of the bony defect. Anand, et al. ${ }^{10)}$ reported the method of covering the defect by using a polypropylene (Medpore) implant in the case when the defect was big (sized over $5 \mathrm{~mm}$ ). Park, et al. ${ }^{7)}$ used titanium mesh for the repair of the bony defect and confirmed that the TMJ tissue did not herniate into the EAC regardless of jaw movement at the postsurgical state. When there is a salivary fistula formation through foramen of Huschke, ligation and then dividing of the fistula tract may be sufficient for treatment of salivary otorrhea from the EAC. ${ }^{11)}$ According to the analysis of Moriyama, et al. ${ }^{8)}$ in which 14 published cases were collected, in 7 cases out of all the surgical treatments, they could observe symptom improvement, and they confirmed that they could achieve satisfactory results without postsurgical complications. However, there is insufficient data on complications of surgical treatment of persistent foramen of Huschke and on longterm progress. Possible postsurgical complications are inserted cartilage movement, TMJ damage, middle ear damage, and mouth opening limitation. ${ }^{10)}$

In this case, because the patient had no intention of being treated surgically due to his weak physical condition from the cerebral infarction, and because the THI score was 18, indicating he was not suffering too much from the clicking tinnitus, we chose conservative treatment. We prescribed an anxiolytic (clonazepam) and a muscle relaxant (eperisone $\mathrm{HCl}$ ) for 2 weeks, and during the follow-up, we could observe neither change in the tinnitus on measurement with a stethoscope nor change in the subjective tinnitus loudness. Therefore, this case is now under progress observation.

We diagnosed objective tinnitus occurring in association with persistent foramen of Huschke. We think this case was very meaningful as a case of objective tinnitus originating clinically very rare cause. Though we failed to obtain full recovery from the tinnitus using conservative treatment, we were able to find a cause of the tinnitus which will be helpful for improving our understanding of tinnitus patients. According to the literature, tinnitus can be successfully treated by surgery. Therefore, we want to emphasize the importance of taking a careful history and performing a physical examination that are appropriate for checking the masticatory movements, as well as describe the related EAC findings, and the symptom occurrence in this disorder, to find out the cause of the objective clicking tinnitus.

\section{REFERENCES}

1) Selesnick SH, Carew JF, DiBartolomeo JR. Herniation of the temporomandibular joint into the external auditory canal: a complication of otologic surgery. Am J Otol 1995;16:751-7.

2) Heffez L, Anderson D, Mafee M. Developmental defects of the tympanic plate: case reports and review of the literature. J Oral Maxillofac Surg 1989;47:1336-40.

3) Yoo HJ, Park SN, Kim DK, Park KH, Kim MJ, Kim JE, et al. Incidence and clinical characteristics of patients with tinnitus according to diagnostic classification. Korean J Otorhinolaryngol-Head Neck Surg 2011;54:392-8.

4) Anson BJ, Donaldson JA. Surgical Anatomy of the Temporal Bone and ear. 3rd ed. Philadelphia: W.B. Saunders Company;1981. p.11423.

5) Lacout A, Marsot-Dupuch K, Smoker WR, Lasjaunias P. Foramen tympanicum, or foramen of Huschke: pathologic cases and anatomic CT study. AJNR Am J Neuroradiol 2005;26:1317-23.

6) Wang RG, Bingham B, Hawke M, Kwok P, Li JR. Persistence of the foramen of Huschke in the adult: an osteological study. J Otolaryngol 1991;20:251-3.

7) Park YH, Kim HJ, Park MH. Temporomandibular joint herniation into the external auditory canal. Laryngoscope 2010;120:2284-8.

8) Moriyama M, Kodama S, Suzuki M. Spontaneous temporomandibular joint herniation into the external auditory canal: a case report and review of the literature. Laryngoscope 2005;115:2174-7.

9) Kryzer TC, Lambert PR. Herniation of temporomandibular joint contents into the external ear canal. Otolaryngol Head Neck Surg 1992;107:607-8.

10) Anand VT, Latif MA, Smith WP. Defects of the external auditory canal: a new reconstruction technique. J Laryngol Otol 2000;114: 279-82.

11) De Zoysa N, Vasani S, Kaniyur S, Frosh A. Gustatory otorrhoea: a rare case of congenital external ear salivary fistula. J Laryngol Otol 2009;123:1371-4. 